\title{
Research on Power System Relay Protection Method Based on Machine Learning Algorithm
}

\author{
Jingying Fang ${ }^{1 *}$, Xiangyun Zhang ${ }^{2}$ \\ ${ }^{1}$ School of Automation, Central South University, Changsha, Hunan, 410083, China \\ ${ }^{2}$ Department of Software Engineering, Harbin University of Science and Technology Rongcheng Campus, Rongcheng, Shandong, \\ 264300, China
}

\begin{abstract}
With the development of power industry, there are gradually high permeability distributed energy systems. However, the existing relay protection is difficult to be effectively applied in this type of power system. To solve this problem, this paper applies machine learning algorithm to power system relay protection. Firstly, the structure of power system with high permeability and distributed energy is analyzed, and the challenges which current relay protection algorithms faced are introduced in detail. Then, the artificial intelligence algorithm is introduced, and the machine learning algorithm in artificial intelligence algorithm and its application in power system are mainly studied. Finally, the power system relay protection based on machine learning algorithm is deeply studied, and the specific implementation method and implementation flow are designed. The machine learning algorithm studied in this paper is helpful to the development of technology in the field of power system relay protection.
\end{abstract}

\section{Introduction}

After the distributed energy is connected to the power system, the original one-way power flow mode has changed into two-way power flow mode, which has changed the structure and power flow of the power system. Especially when the distributed energy is highly permeated into the power system, the operation mode of the power system is more complex, and the traditional relay protection method can not be fully applied to the power system with distributed energy[1-2]. In view of the fact that the traditional protection algorithm is difficult to apply to the development of power system, this paper applies machine learning algorithm to the relay protection of power system.

\section{New Problems of Relay Protection}

\subsection{Distributed Energy}

With the continuous depletion of coal resources and pollution problems caused by coal power generation, new energy generation such as photovoltaic and wind power has become a green energy mode with potential for development, which has been paid more and more attention by various countries. Depending on their geographical and climatic advantages, various forms of new energy generation can be developed according to local conditions, and connected to the main power grid to complement each other[3].
Due to the limitation of power and voltage levels, new energy generation usually forms a micro-grid and is connected to the distribution network by a contact switch, as shown in Figure 1. New energy sources such as micro gas turbines, wind power and photovoltaic power generation often produce direct current electric energy, which is not completely matched with the current distribution network. Therefore, it is necessary to convert the electric energy into the matching electric energy with the access distribution network through the power electronic components as the core of the power converter.

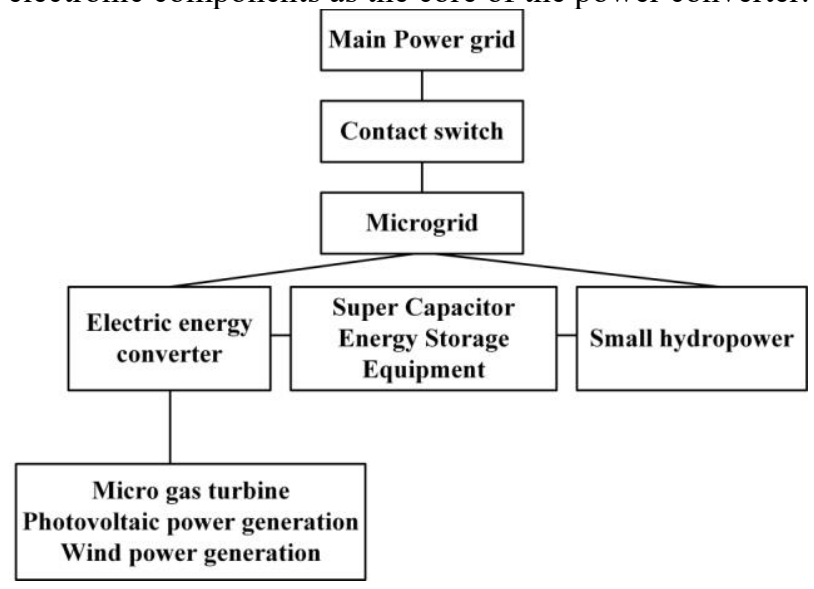

Figure 1. Power system structure with distributed energy

Because the new energy generation is easy to be constrained by the natural environment, it has the characteristics of instability, intermittence and fluctuation[4]. Usually it is equipped with energy storage equipment such as supercapacitors, which absorbs excess 
power when the power is surplus, and provides power when the power is insufficient, so as to maintain the stability of the power system. Traditional main power grid has relatively fixed operation mode, and the size and direction of power flow are easy to determine, which is conducive to the realization of power system relay protection. The new energy is connected to the main power grid in the form of micro-grid, which makes the operation mode of power system more complex, increases the randomness and uncertainty of power flow distribution, and makes the traditional relay protection method of power system not necessarily fully applicable, so a new method is needed for in-depth analysis.

\subsection{Challenges of Relay Protection Algorithms}

Distributed energy is connected to the main grid by high permeability, which makes the protection mode of power system more complex. The improvement measures of relay protection can help to reduce the difficulty of relay protection, but it can not completely eliminate the impact of distributed energy on traditional relay protection, mainly in the following aspects.

1)The short-circuit current in the network will decrease or increase with the access of distributed generation, which may lead to the maloperation, rejection or loss of selectivity of protection in the distribution network.

2)For the small current grounding system, the gridconnected transformer should adopt $\Delta / \mathrm{Yg}$ connection mode, in which the system side is $\Delta$ connection mode; for the small resistance grounding system, the grid-connected transformer should adopt $\mathrm{Yg} / \Delta$ connection mode, in which the system side is Yg connection mode. For low current grounding system, the access of distributed generation has no effect on zero sequence protection based on steadystate characteristic fault detection algorithm.

3)The type of distributed generation, short circuit capacity, grid-connected position and neutral grounding impedance of grid-connected transformer are all important factors affecting the protection of distribution network. The main power grid with distributed energy has changed the power distribution and impedance distribution of the traditional power grid. The classical relay protection method is difficult to fully adapt to the relay protection with distributed energy. It is urgent to introduce advanced algorithms such as artificial intelligence into complex applications such as relay protection of power system with distributed energy to improve the accuracy of protection.

\section{Machine Learning Method}

\subsection{Artificial Intelligence Algorithms}

The term artificial intelligence has been proposed since 1956, and the emergence of electronic computers as a tool to simulate human thinking has enabled the artificial intelligence technology to have a platform for its exploration and development. Artificial intelligence technology is an innovative technology leading the future.
With the development of national economy, internet, big data and supercomputer, the development of artificial intelligence technology has entered a new stage featuring in-depth learning, cross-border integration, man-machine cooperation and self-control of group intelligence development. These artificial intelligence technologies with new characteristics will have a significant and farreaching impact on human production, life and even thinking mode.

\subsection{Machine Learning Algorithms and Their Electric Power Applications}

As the core of artificial intelligence, machine learning (ML) has been applied more and more in dealing with complex systems. Artificial intelligence algorithms such as machine learning specialize in how computers simulate or implement human learning behavior in order to acquire new knowledge or skills, reorganize existing knowledge structures and continuously improve their performance. Machine learning relies on good data sources and needs good data support. At present, with the rapid development of large data and super-performance computer technology, machine learning has improved a larger number of better training methods, which is conducive to the use of machine learning to achieve complex power advanced applications [5]. This paper designs an advanced application of power machine learning algorithm based on power big data support platform. With the help of large power data, it can provide data source for machine learning, so that advanced applications of power system such as effective relay protection can be obtained through machine learning algorithm [6].

With the development of smart grid, more sensor elements and various types of smart electronic devices, smart meters are equipped in the power field. The smart electronic devices and smart meters collect real-time data of current, voltage and switching status in the power operation site. After pretreatment, the collected data will be sent to the large data support platform of power through the communication network. The power big data support platform first carries on the power big data collection, the power big data analysis and the power big data storage. On the one hand, the stored large power data are stored in the knowledge database, and the functions of query, record and diagnosis of the knowledge database are provided. On the other hand, the collection and analysis results of the large power data are imported into the machine learning algorithm, and the advanced application functions of the power system are completed by combining the experience provided by the knowledge database.

\section{Power system relay protection based on machine learning algorithm}

\subsection{Implementation Method}

Power system relay protection based on machine learning algorithm can give full play to the advantages of machine 
learning in dealing with the diversity and complexity of power system relay protection with distributed energy. Machine learning algorithm collects training data of relay protection called from knowledge database and real-time operation data obtained from relay protection field.

The algorithms of probability theory, statistics, approximation theory, convex analysis and algorithm complexity theory are used to train power system model. The fault characteristics of power system with distributed energy are analyzed in detail, as shown in Figure 2.

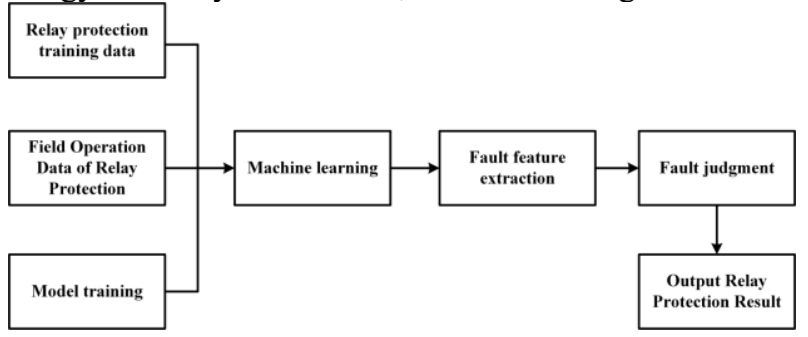

Figure 2. Application of Machine Learning Algorithms in Power System Relay Protection

\subsection{Implementation Flow}

The application process of power system relay protection based on machine learning algorithm is shown in Figure 6. Firstly, it collects information related to relay protection such as electric quantity and switch quantity of power system, pre-processes information, and eliminates the misinformation generated during field transmission or communication network transmission. Then, it is judged whether the collected information is sufficient for the machine learning algorithm, and if the amount of data is not enough, the relay protection information required by the machine learning algorithm is continuously collected; once the relay protection information required by the machine learning algorithm is sufficient, the The machine learning algorithm is performed to extract the power failure characteristics required for relay protection, and further to identify the relay protection. Finally, it is judged whether the power system has a fault, and if there is no fault, it returns; if there is a fault, the relay protection action such as tripping or alarm is taken.

\section{Conclusion}

With the distributed energy and high permeability main grid, the operation of power system becomes more complex and the relay protection is more difficult. In this paper, the machine learning method is applied to the relay protection of power system, and the large data method is used to provide data support for machine learning, so as to achieve effective and reliable relay protection of power system. The main contents are as follows.

1) The structure of power system with distributed energy is introduced, and the challenges faced by traditional relay protection algorithms are studied.

2) Analyse the characteristics of artificial intelligence algorithm, especially machine learning algorithm, and study the application of machine learning algorithm in power system.
3) The relay protection of power system based on machine learning algorithm is deeply studied, and the realization method and process are introduced in detail.

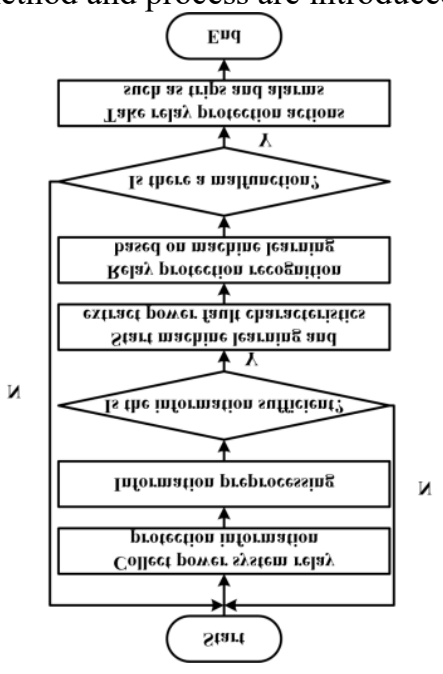

Figure 3. Implementation process

\section{References}

1. Blaabjerg, F., Teodorescu, R., Liserre, M., Timbus, AV. (2006) Overview of control and grid synchronization for distributed power generation systems. IEEE T IND ELECTRON., 53(5): 13981409.

2. Carrasco, JM., Franquelo, LG., Bialasiewicz, JT., Galvan, E., Portillo, R., Prats, MM., Leon, JI., Moreno-Alfonso, N. (2006) Power-electronic systems for the grid integration of renewable energy sources: A survey. IEEE T IND ELECTRON., 53(4): 1002-1016.

3. Lopes, JAP., Hatziargyriou, N., Mutale, J ., Djapic, P., Jenkins, N. (2007) Integrating distributed generation into electric power systems: A review of drivers, challenges and opportunities. ELECTR POW SYST RES., 77(9): 1189-1203.

4. Huang, AQ., Crow, ML., Heydt, GT., Zheng, JP., Dale, SJ. (2007) The Future Renewable Electric Energy Delivery and Management (FREEDM) System: The Energy Internet. P IEEE., 99(1): 133148.

5. Wan, C., Xu, Z., Pinson, P., Dong, ZY., Wong, KP. (2014) Probabilistic Forecasting of Wind Power Generation Using Extreme Learning Machine. IEEE T POWER SYST., 29(3): 1033-1044.

6. Morales, JA., Orduna, E., Rehtanz, C. (2014) Classification of lightning stroke on transmission line using multi-resolution analysis and machine learning. INT J ELEC POWER., 58: 19-31. 